\title{
Loron (Tar-Vabriga, Croatie)
}

Campagne de fouilles 2011

\section{Corinne Rousse et Francis Tassaux}

Musée régional de Poreč (Croatie), Ausonius (Université de Bordeaux 3), École française de Rome et Centre Camille Jullian (Université Aix-Marseille / CNRS) (éd.)

\section{(2) OpenEdition Journals}

Édition électronique

URL : http://journals.openedition.org/cefr/217

DOI : $10.4000 /$ cefr. 217

ISSN : 2282-5703

Éditeur

École française de Rome

Référence électronique

Corinne Rousse et Francis Tassaux, «Loron (Tar-Vabriga, Croatie) », Chronique des activités archéologiques de l'École française de Rome [En ligne], Balkans, mis en ligne le 19 décembre 2012, consulté le 18 octobre 2019. URL : http://journals.openedition.org/cefr/217 ; DOI : 10.4000/cefr.217

Ce document a été généré automatiquement le 18 octobre 2019

(c) École française de Rome 


\title{
Loron (Tar-Vabriga, Croatie)
}

\author{
Campagne de fouilles 2011
}

\section{Corinne Rousse et Francis Tassaux}

Musée régional de Poreč (Croatie), Ausonius (Université de Bordeaux 3), École française de Rome et Centre Camille Jullian (Université Aix-Marseille / CNRS) (éd.)

1 La mission franco-croate intervient sur le site de Loron dans le cadre d'un accord quadripartite associant le Musée territorial de Poreč (Croatie - V. Kovačić), le centre Ausonius de l'université de Bordeaux 3, l'école française de Rome et l'université de Padoue. Elle est financée pour la partie française par le ministère des Affaires étrangères et européennes et l'École française de Rome, dans le cadre d'un programme quadriennal intitulé L'Istrie et la mer. En 2011, le centre C. Jullian (CNRS - Université d'Aix Marseille) a également apporté une contribution financière et scientifique à la mission. Enfin, nos partenaires croates, la municipalité de Tar-Vabriga, l'office du tourisme de la commune de Tar-Vabriga (Turistička Zajednica Tar-Vabriga) et le ministère croate de la recherche participent au financement, à la logistique et à la valorisation des fouilles.

2 Dernière année du quadriennal de recherche (MAE-EFR), la campagne 2011 marque la fin de l'étude consacrée à l'atelier d'amphores, avec la fouille de l'aile occidentale de l'atelier qui restait le seul édifice non encore dégagé (fig. 1). On rappellera que ce complexe artisanal fait partie d'un vaste ensemble édifié ex nihilo au bord de la mer vers 10 ap.J.-C., caractérisé par la fonctionnalité des espaces de production, organisés autour d'une vaste cour centrale (espace 37) dotée d'une réserve d'eau avec fontaine (espace 47). Quatre fours à amphores, parfaitement alignés, occupent la pièce centrale de l'édifice nord (espace 45), entourés par deux vastes préaux de séchage (espaces 48-49). À l'extrémité de l'édifice oriental, une autre pièce accueille une batterie de petits fours servant probablement à la cuisson de céramiques communes (espace 36). Cette organisation rationnelle, régie par un fort principe de symétrie, suggère qu'un plan d'architecte ait dirigé la construction de l'atelier. Il subit peu de modifications jusqu'à l'arrêt des activités artisanales dans la deuxième moitié du IV $\mathrm{s}$. ap. J.-C. L'occupation du site se poursuit jusqu'au milieu du V $\mathrm{V}^{\mathrm{e}}$ s. ap. J.-C., suivie d'un abandon complet et d'un arasement général des édifices. 
Fig. 1 - Loron. Planimétrie du complexe artisanal et relevé complet réalisé en 2011.

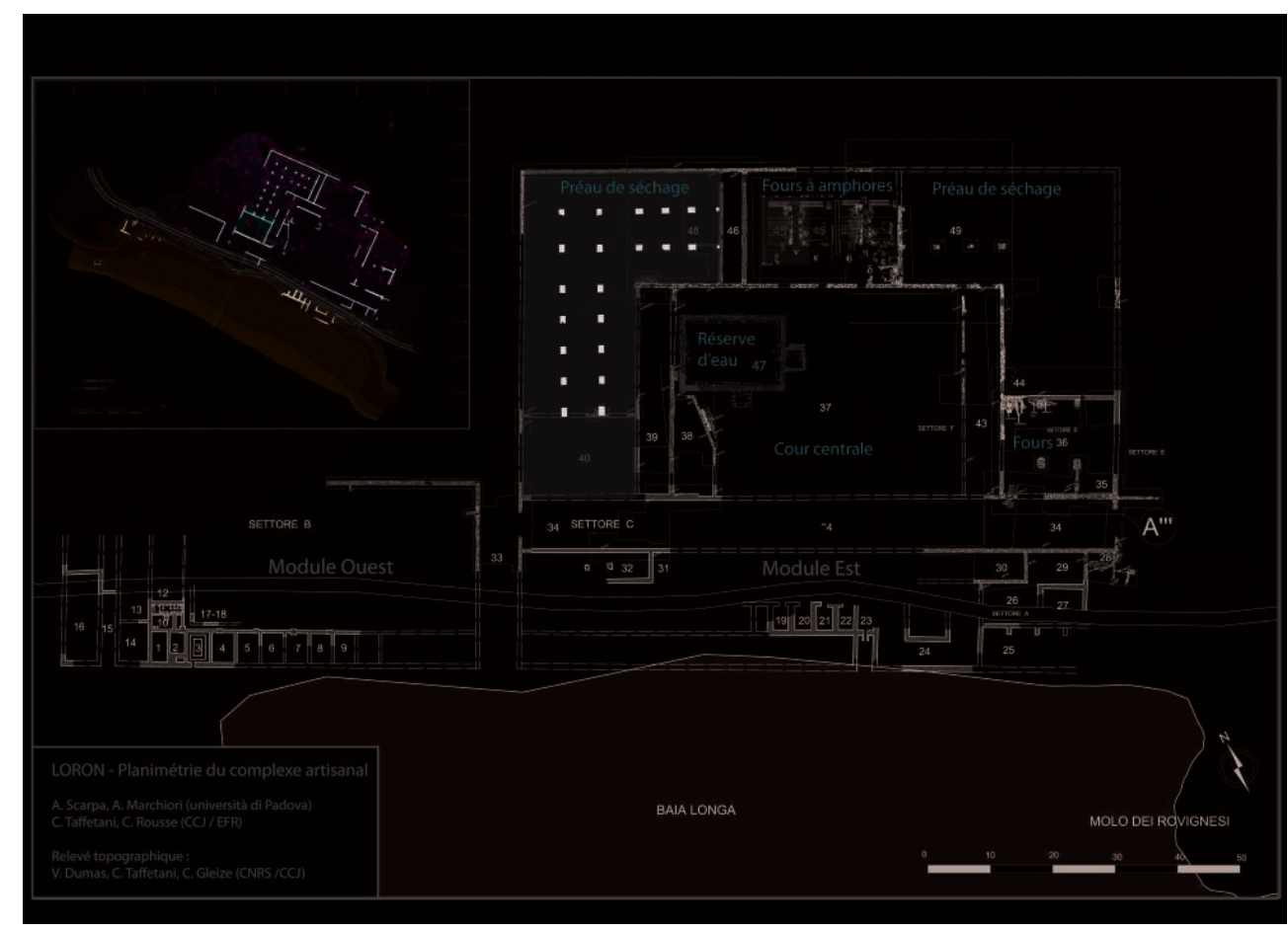

En grisé, le secteur d'intervention dans l'aile ouest de l'atelier d'amphores.

3 Centrée sur l'édifice ouest, la mission 2011 a permis d'étudier dans le détail l'architecture des préaux de séchages (espace 48) et la circulation au sein de l'atelier (espace 40). Aucune structure artisanale n'a pu être retrouvée dans l'espace 40, qui ferme le bâtiment au sud. En revanche, la découverte de trois sépultures en amphores dans les strates d'abandon documente pour la première fois les derniers horizons d'occupation dans le secteur occidental.

La fouille a été conduite sous la direction de Corinne Rousse, avec la collaboration de trois chefs de secteurs Audrey Bertrand (École française de Rome), Giuseppe Silvestri (Rome) et Valerio De Leonardis (Université de Roma 3), de Claudio Taffetani (Université d'Aix Marseille-Roma 3) pour la topographie et Julie Marangoni (Université de Bordeaux 3) pour l'enregistrement du mobilier. Cinq étudiants des universités d'Aix Marseille (Jonathan Devogelaere, Regis Maggiori), de Bordeaux 3 (Julie Bernini, Solène Rousseau) et de Budapest (Anna Nagy) ont également participé à la mission, ainsi que Laurence Marlin (Attachée de conservation du patrimoine - ministère de la Culture) et Élise Fovet (post-doctorante, Université de Franche Comté). Paola Maggi (Université de Trieste) et Yolande Marion (Ausonius - Université de Bordeaux 3) ont assuré une mission d'inventaire et d'étude du mobilier.

5 À la campagne de fouille ont été adossées une mission de relevés topographiques d'ensemble (fig. 1-2) réalisée par Vincent Dumas (CNRS - Centre C. Jullian) et Claudio Taffetani (université d'Aix Marseille-Roma $3 /$ Centre C.Jullian), ainsi que des campagnes de prospections terrestres (Élise Fovet) et géophysiques (Myriam Schmutz et Amélie Blondel: EGID-Université de Bordeaux 3). Leurs objectifs étaient d'approfondir la connaissance de la topographie du promontoire de Loron et de son 
potentiel archéologique, notamment la localisation de la villa maritime contrôlant ce grand domaine sénatorial et impérial (fig. 2).

Fig. 2 - Loron. Le domaine aristocratique entre les baies de Červar Porat au sud et de Santa Marina au nord.

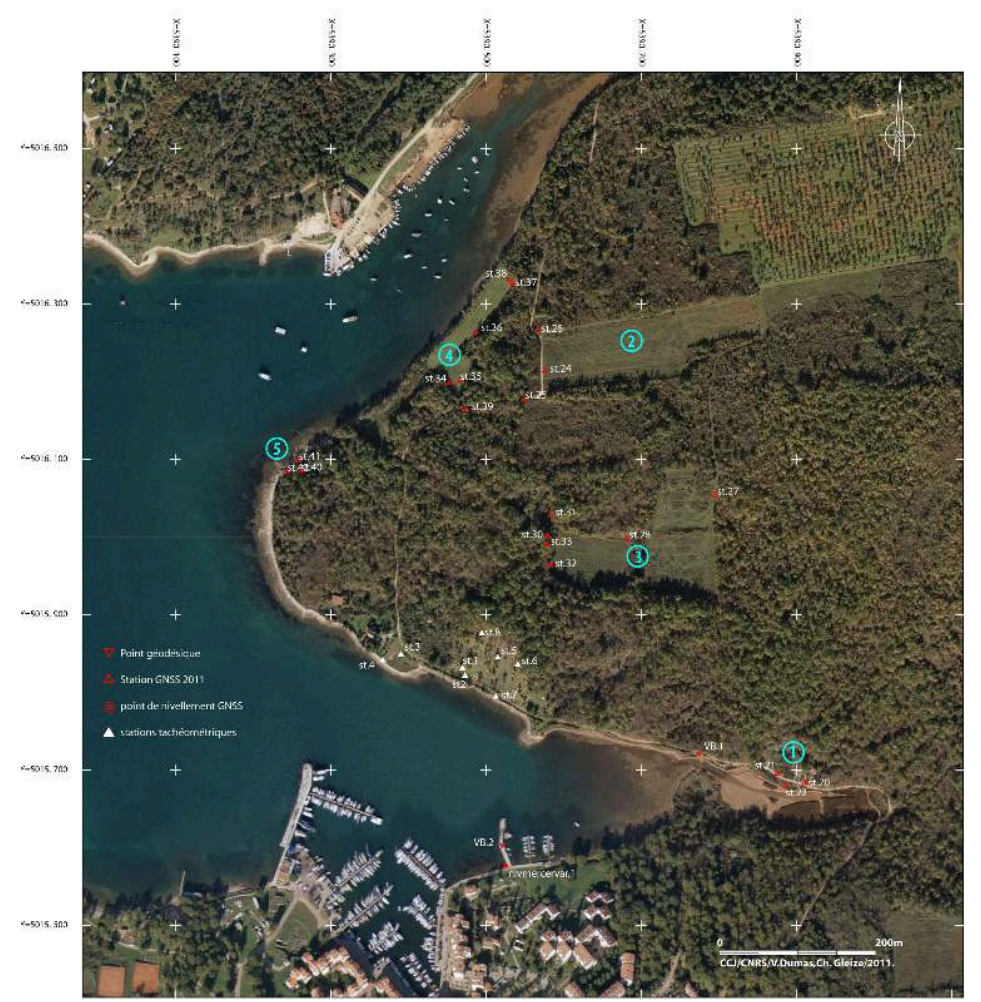

Stations topographiques implantées pour l'étude.

V. Dumas et Ch. Gleize, CNRS / CCJ.

\section{L'aile occidentale de l'atelier}

6 Accolée à l'édifice nord, l'aile occidentale mesure $38 \mathrm{~m}$ de long pour $13,5 \mathrm{~m}$ de large (fig. 3). Elle est constituée de deux pièces, séparées par un mur interne (MR 3230) : au sud, un espace fermé dont la fonction est probablement artisanale (espace 40) ; au nord, un vaste espace de stockage qui se poursuit sur l'édifice nord, présentant un plan à trois nefs et interprété comme un préau de séchage (espace 48 ). 
Fig. 3 - Loron. Planimétrie de l'aile ouest de l'atelier.

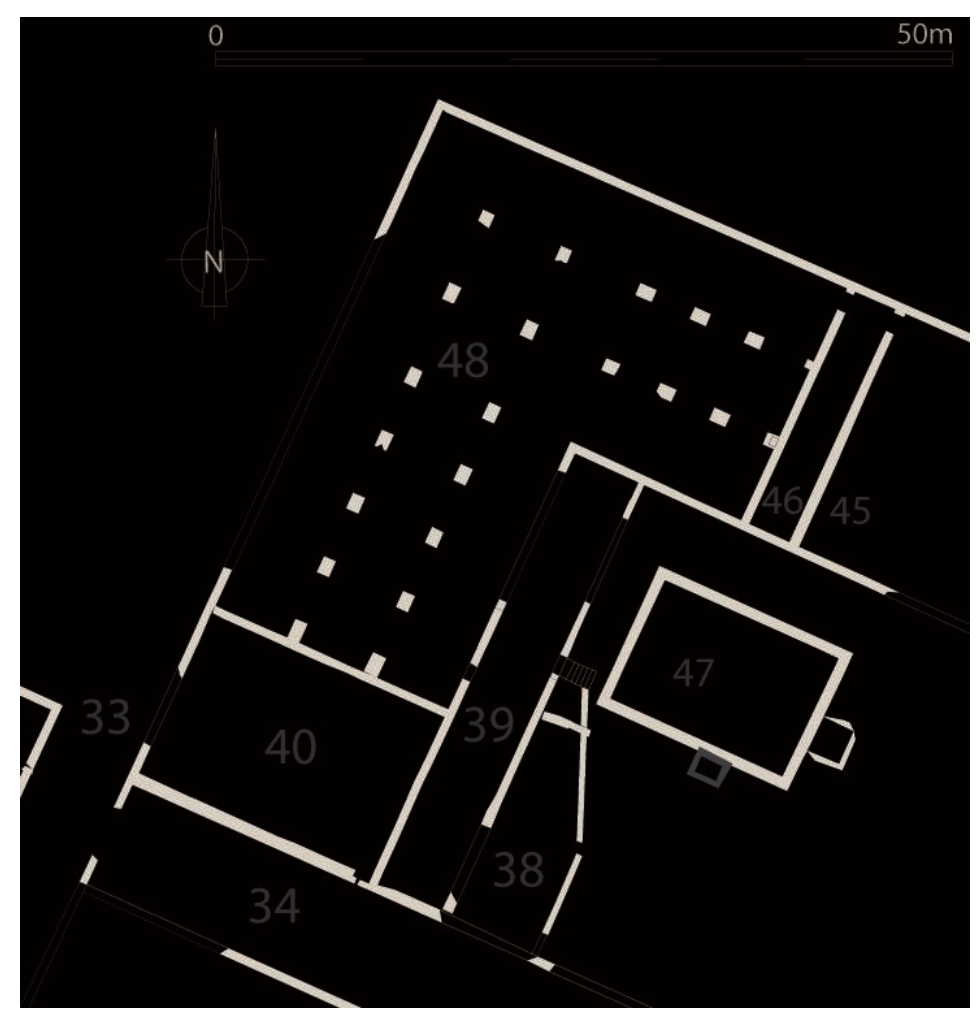

C. TAFFETANI, CCJ / EFR.

\section{L'espace 40}

7 L'espace 40 est une vaste pièce rectangulaire de $18,5 \mathrm{~m}$ sur $13,5 \mathrm{~m}\left(230 \mathrm{~m}^{2}\right)$ à l'extrémité sud de l'aile occidentale de l'atelier (fig. 4). Il est délimité à l'ouest et au sud par des murs de façade donnant sur des espaces de circulation majeurs : une voie nord-sud descendant vers la mer et un long passage est-ouest qui dessert les édifices artisanaux et la cour. Les murs nord (MR 3230) et est (MR 3125) séparent la pièce du préau de séchage (48) et d'une étroite zone de stockage (espace 39) donnant sur la cour.

Tous les murs sont fortement spoliés, avec un arasement en gradin qui suit la pente générale du terrain. Ils ont été dégagés sur toute leur longueur, révélant la présence d'un seuil bien conservé dans l'angle sud-est de la pièce (fig. 5). Cette ouverture donnait accès à la voie interne qui longe au sud l'atelier. Sa position répond exactement à l'emplacement choisi pour implanter le seuil de l'espace 36 , vaste pièce dotée de petits fours à l'extrémité de l'édifice oriental. La disposition en miroir des accès aux deux ailes entourant la cour souligne le principe de symétrie qui organise, jusque que dans le détail des ouvertures, l'ensemble des édifices de l'atelier.

9 Une série de spoliations plus profondes observées à intervalle régulier sur la façade sud de l'espace 40 suggère la récupération d'éléments de décor qui ornaient la façade sud de l'aile ouest, donnant sur la voie interne. Il s'agit peut-être de colonnes en calcaire, dont une des bases a été retrouvé dans les niveaux de remblais tardifs, remployée comme meule. En outre, un décor architectural à colonnes, en calcaire et en brique, est déjà attesté sur les façades des édifices est et ouest donnant sur la cour ${ }^{1}$. 
Fig. 4 - Loron. Espace 40. Vue du sud.

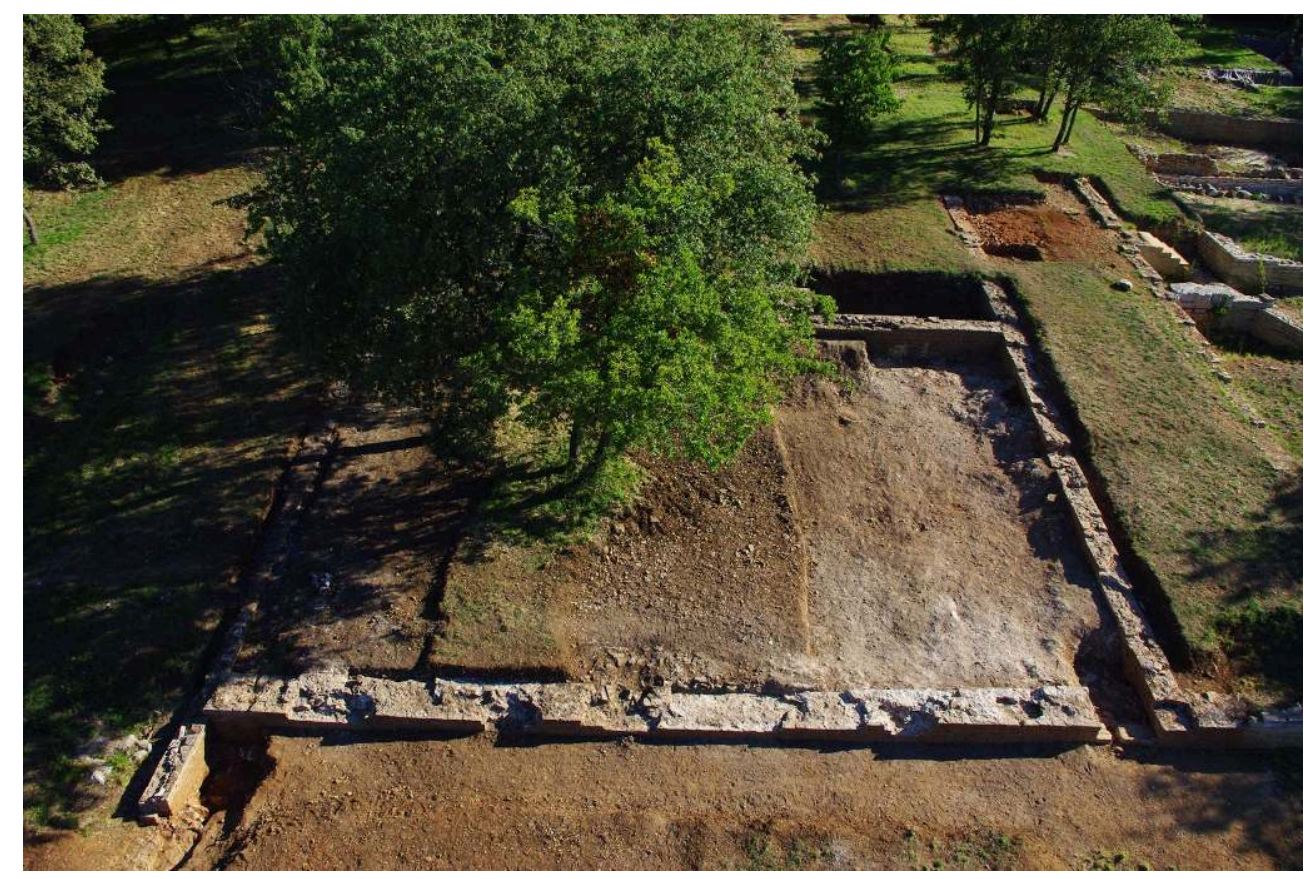

CLICHÉ C. TAFFETANI, EFR.

Fig. 5 - Loron. Espace 40. Seuil.

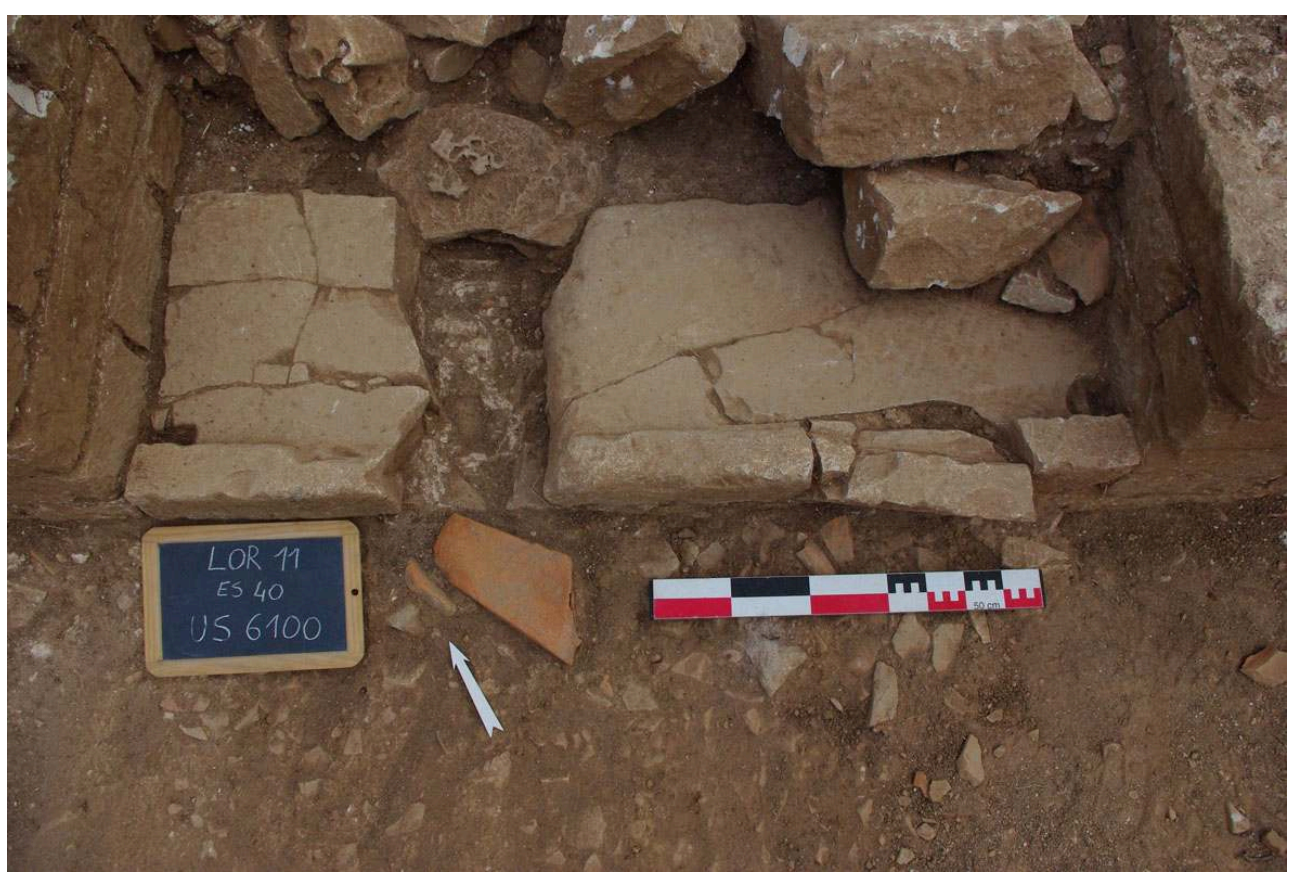

Cliché A. Bertrand, EFR.

L'étude de la stratigraphie à l'intérieur de l'espace 40 s'est révélée décevante, en dépit des moyens mis en œuvre pour dégager un peu plus d' $1 / 3$ de la pièce (fouille manuelle et décapages à la pelle mécanique). Aucune trace d'installations artisanales ou d'éléments architecturaux internes n'a pu être retrouvée. Des sondages profonds 
réalisés aux angles sud-est et nord-est de la pièce montrent que les niveaux de circulation ne sont pas conservés. Ne subsiste donc qu'une couche de destruction hétérogène associée à la spoliation des élévations, datée par le mobilier de la deuxième moitié du IV $\mathrm{IV}^{\mathrm{e}}$-milieu $\mathrm{V}^{\mathrm{e}} \mathrm{s}$. ap. J.-C. Elle est recouverte par des remblais plus tardifs correspondant à l'abandon et à l'arasement général du complexe dans la deuxième moitié du V s. ap. J.-C.

\section{Les contextes funéraires tardifs}

11 La découverte de sépultures dans l'espace 40 a été une surprise, car les données rassemblées jusqu'ici dans le secteur occidental de l'atelier tendaient à montrer un abandon complet des édifices dans la deuxième moitié du IV $\mathrm{e}$ s. ap. J.-C. Jusqu'à cette campagne, nous pensions à un repli de l'occupation sur le secteur oriental, l'ouest de l'atelier étant alors soumis à d'importantes interventions de spoliation, bien notées dans les niveaux d'abandon de la réserve d'eau par exemple. On sait grâce aux précédentes campagnes que les bâtiments artisanaux, encore en fonction au début du $\mathrm{IV}^{\mathrm{e}} \mathrm{s}$. ap. J.-C., sont progressivement défonctionnalisés dans le courant de ce siècle et servent ensuite de carrière de pierre aux derniers habitants du site.

La première sépulture (SP 6025) a été découverte lors de la fouille de la couche de destruction du mur oriental (MR 3125) dans l'angle nord-est de l'espace 40. Orientée nord-sud elle a été installée le long du mur MR 3125 et appuyée à celui-ci (fig. 6). Deux autres sépultures (SP 6085 et SP 6104) ont été découvertes plus au sud, elles-mêmes alignées le long du mur MR 3125. Elles ont été fouillées dans l'urgence, après avoir constaté l'intervention de fouilleurs clandestins sur la première sépulture. Les ossements et prélèvements associés ont été déposés dans les réserves archéologiques du musée de Poreč dans l'attente d'une étude approfondie. Les analyses anthropométriques ont été confiées, à la demande du responsable de fouille V. Kovačić, à $\mathrm{P}$. Raić (Institut d'anthropologie - Zagreb) qui avait déjà réalisé l'étude des individus inhumés dans l'espace 36. 
Fig. 6 - Loron. Planimétrie de l'espace 40 et positionnement des contextes funéraires.

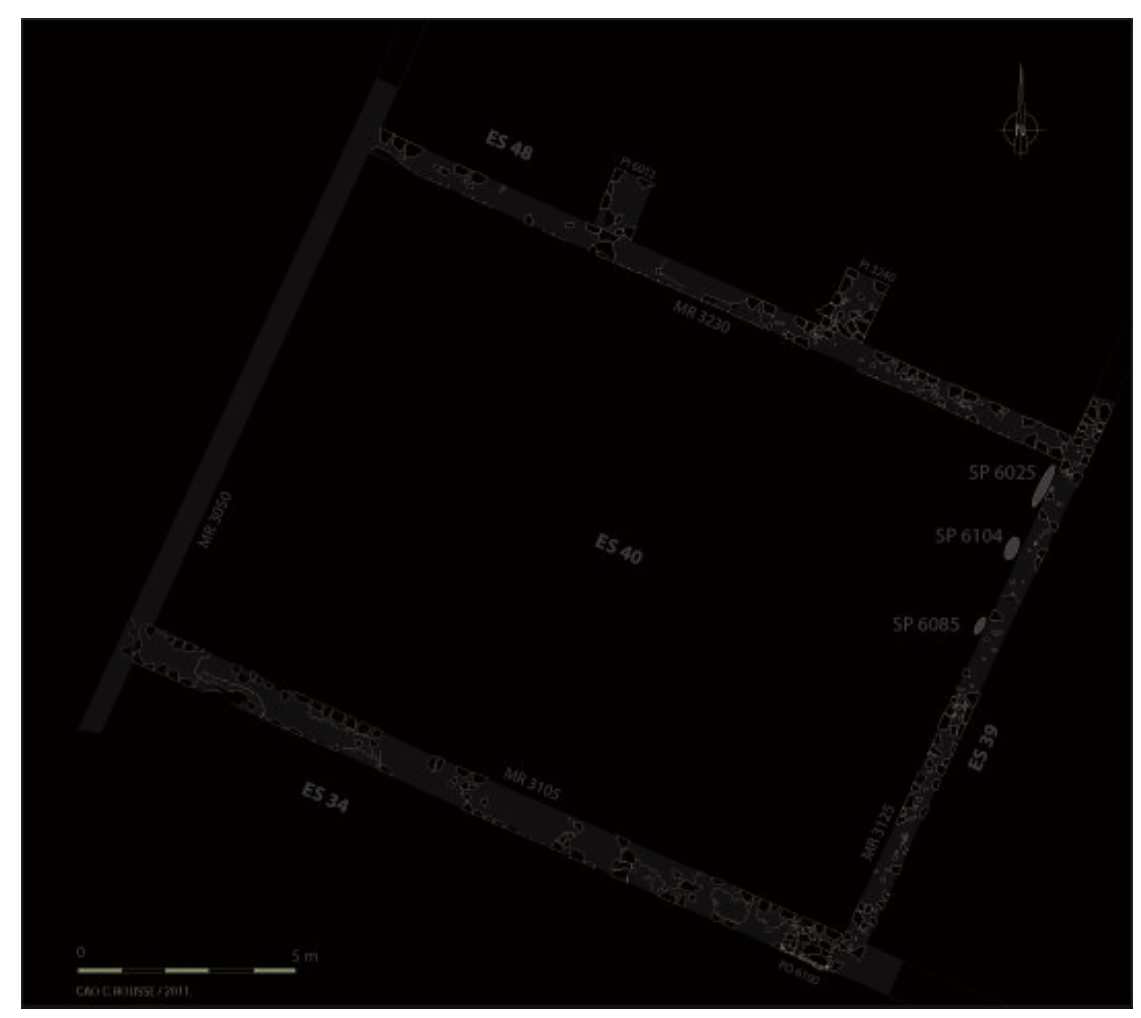

CAO C. Rousse.

13 Ces trois contextes funéraires correspondent à l'inhumation en amphore de nouveaux nés ou de très jeunes enfants. Une première analyse a pu être menée in situ et soumise, pour l'identification des amphores, à l'expertise de M. Bonifay (CNRS-Centre C. Jullian). Elle nécessite d'être confirmée par une étude complète, à la fois des données anthropométriques et du mobilier :

SP 6025 : Sépulture en amphore africaine $(1,1 \mathrm{~m}$ de long, $30 \mathrm{~cm}$ de diamètre) de type indéterminé : il s'agit soit d'un exemplaire de Keay 59/8B diffusée au V $\mathrm{V}^{\mathrm{e}}$ s., soit d'un type africain récemment attesté dans les contextes funéraires tardifs $\left(V^{e} s\right.$.) de la rue Malaval à Marseille ${ }^{2}$ et, beaucoup plus près de Loron, dans les niveaux tardifs de la villa rustica de Skolarice près de Koper (Slovénie), où ce type amphorique a servi de sépulture à un jeune enfant ${ }^{3}$.

L'amphore SP 6025 est disposée nord-sud le long du mur MR 3125, à l'angle nord-est de l'espace 40 (fig. 7). Le défunt est un tout jeune enfant, déposé la tête au sud, soit au fond de l'amphore (fig. 8). Un fragment de verre et un fragment de métal avec décor pourraient correspondre au mobilier funéraire.

16 SP 6085 : Sépulture en amphore africaine de type africaine I (III ${ }^{\mathrm{e}}-\mathrm{V}^{\mathrm{e}} \mathrm{s}$. ap. J.-C.), disposée le long du mur MR 3125, orientée nord-sud. L'amphore est cassée au niveau du col par un sondage superficiel réalisé en 2003. Les ossements se concentrent dans la partie inférieure de l'amphore, au nord. Aucun mobilier funéraire n'a été relevé. Il s'agit d'une sépulture d'un ou deux nouveau-nés, ce que pourra préciser l'analyse anthropologique.

SP 6104 : Sépulture constituée de deux amphores africaines encastrées (fig. 9) : un fond d'amphore africaine de type Keay $62 \mathrm{Q} /$ Albenga 11/12 (deuxième moitié du V $\mathrm{V}^{\mathrm{e}}$ siècle) 
est conservé jusqu'à mi hauteur de la panse (US 6104). L'autre moitié de la sépulture est constituée par la partie supérieure d'une amphore africaine (US 6116) conservée du bord jusqu'à l'épaule, avec une anse entière (fig. 10). Cette amphore est une variante du type indéterminé attesté dans la nécropole de la rue Malaval à Marseille (Bonifay, Capelli, Molliner 2011, fig. 11-12, n. 29), datée de la deuxième moitié du Ve s. ap. J.-C. La sépulture est installée le long du mur MR 3125, orientée nord-sud. Le corps du défunt, probablement un nouveau né ou un très jeune enfant, était soigneusement recouvert par un fond d'assiette en sigillée D africaine de type Hayes 67 (atelier d'El Mahrine : décor de style A(ii)/El Mahrine I.2). Un fragment d'amphore africaine de type " spatheion " protégeait le crâne du défunt. Le mobilier funéraire s'avère plus riche, avec la présence d'une amphorette orientale de type MR3 / LR3, d'un bracelet en bronze décoré, de trois petits coquillages percés pouvant correspondre à un collier et d'une coquille d'huitre, probablement impliquée dans le rituel funéraire. Chronologiquement, $l^{\prime}$ ensemble du mobilier place la réalisation de la tombe dans la deuxième moitié du $V^{\mathrm{e}} \mathrm{s}$. ap. J.-C.

Fig. 7 - Loron. Sépulture en amphore SP 6025.

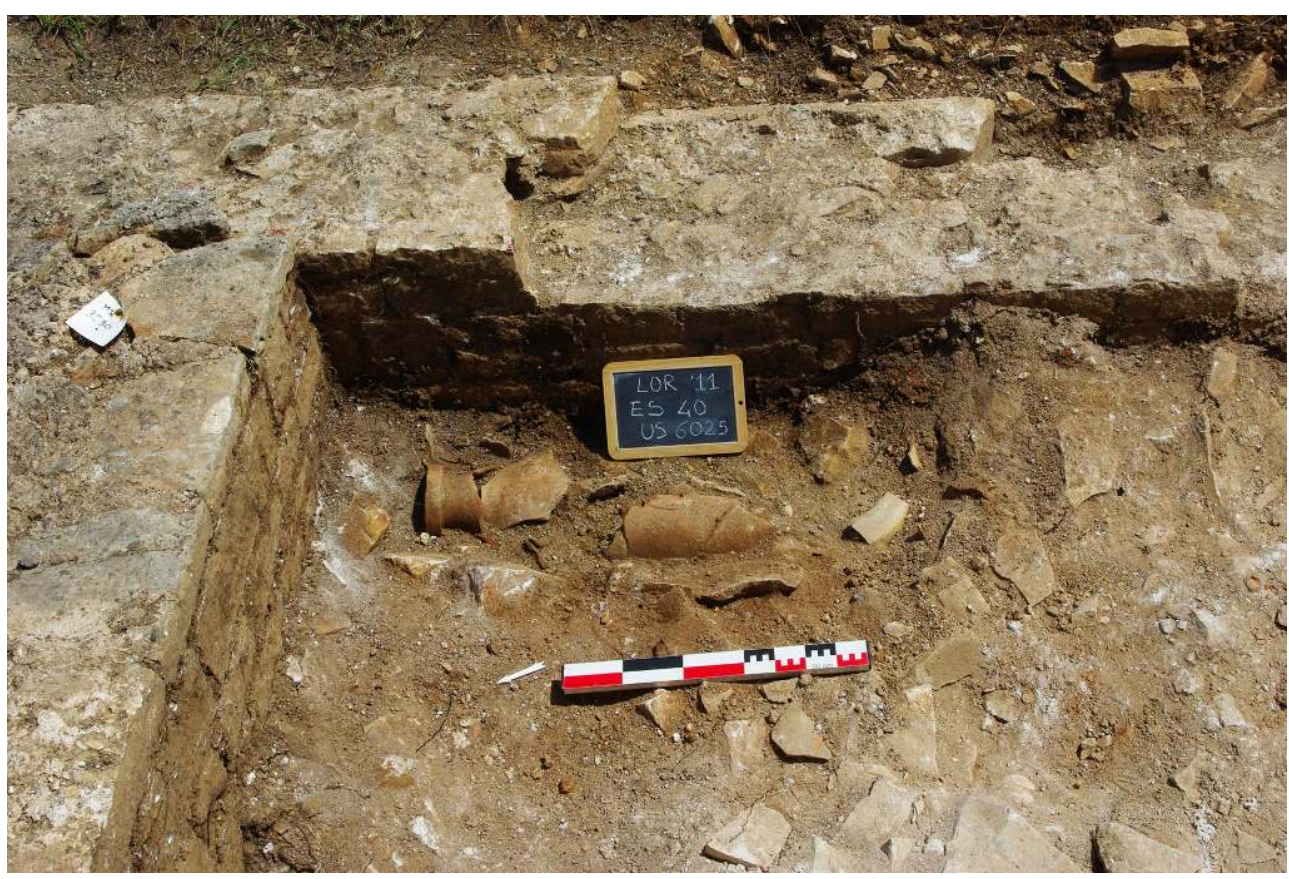

Cliché C. Rousse. 
Fig. 8 : Loron. Sépulture en amphore SP 6025 en cours de dégagement.

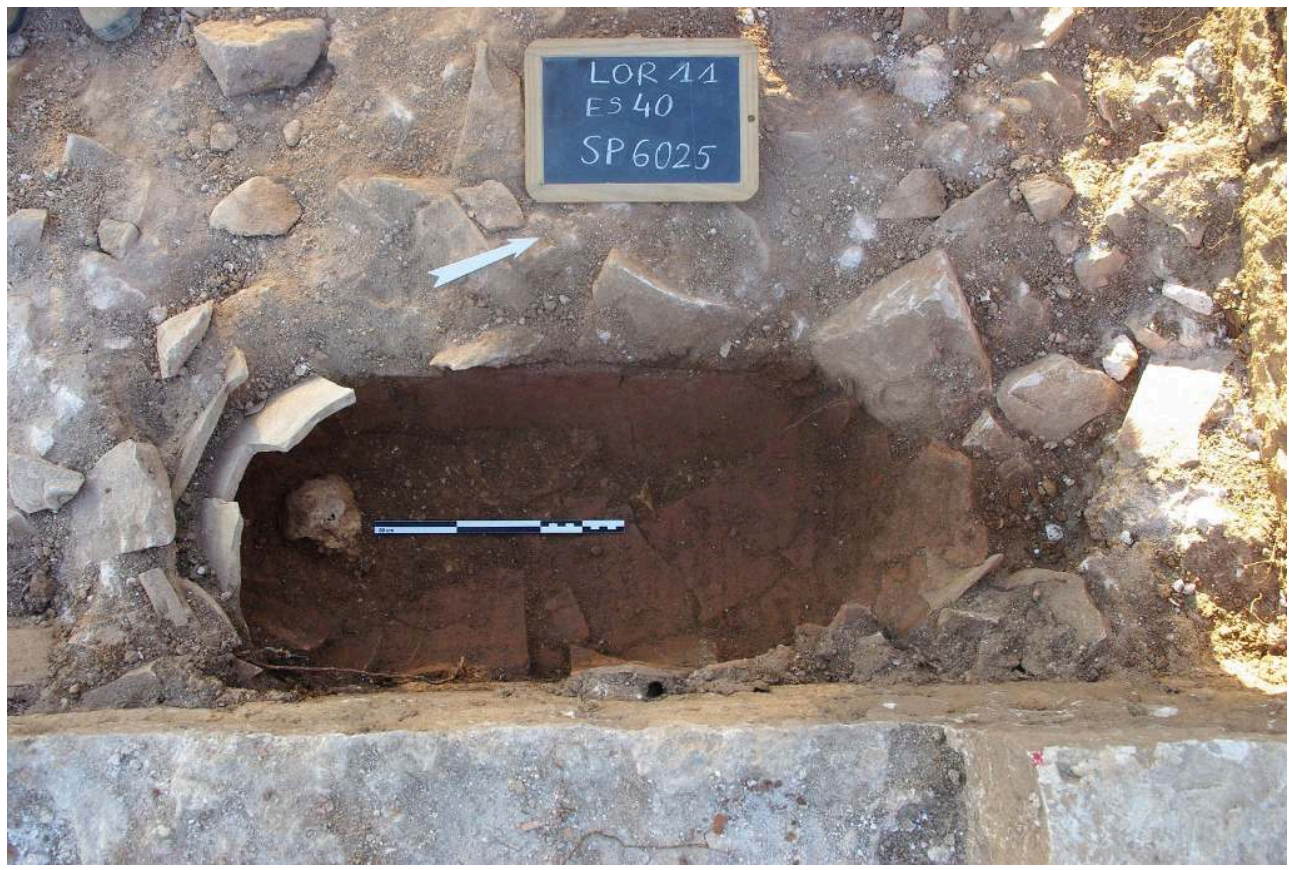

CLICHÉ G. SILVESTRI.

Fig. 9 : Loron. Sépulture SP 6104 avec ses deux amphores encastrées et mobilier funéraire en place.

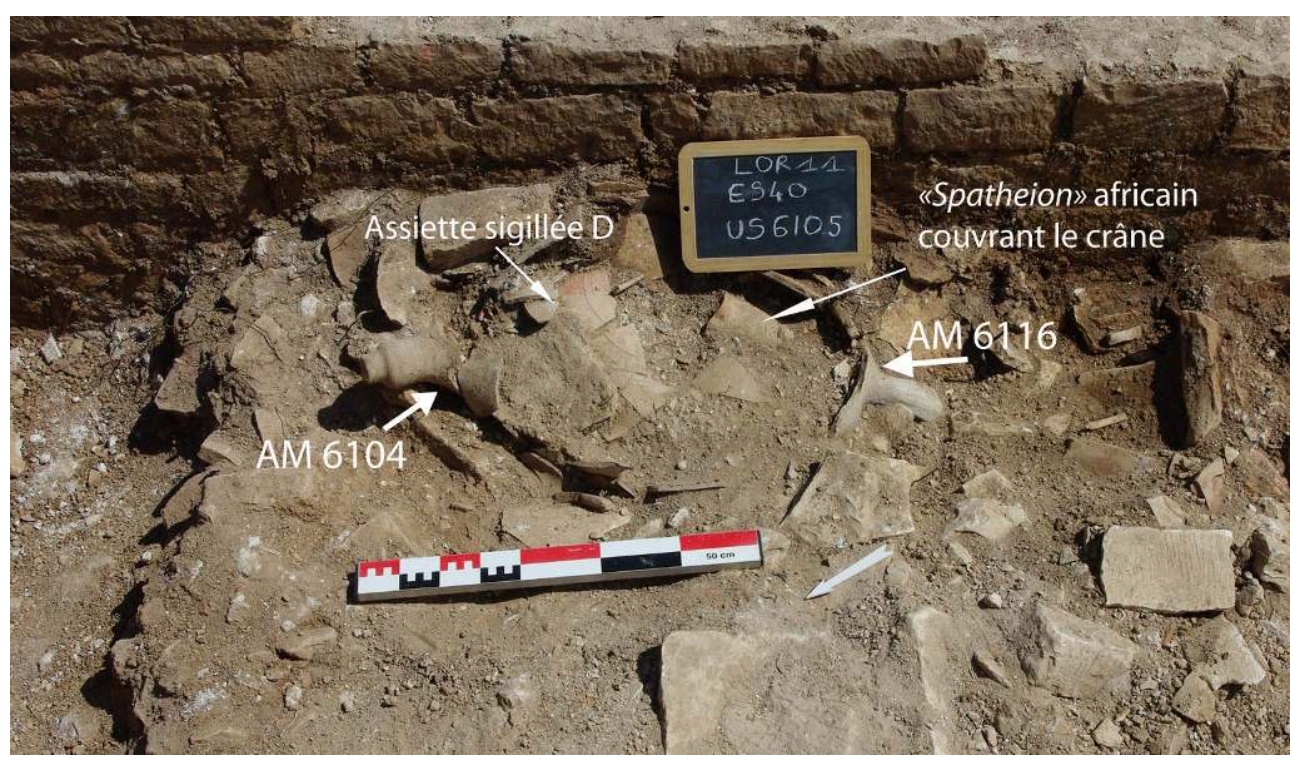

Cliché C. Rousse. 
Fig. 10 : Loron. Sépulture SP 6104 en cours de dégagement, détail de l'amphore AM 6116.

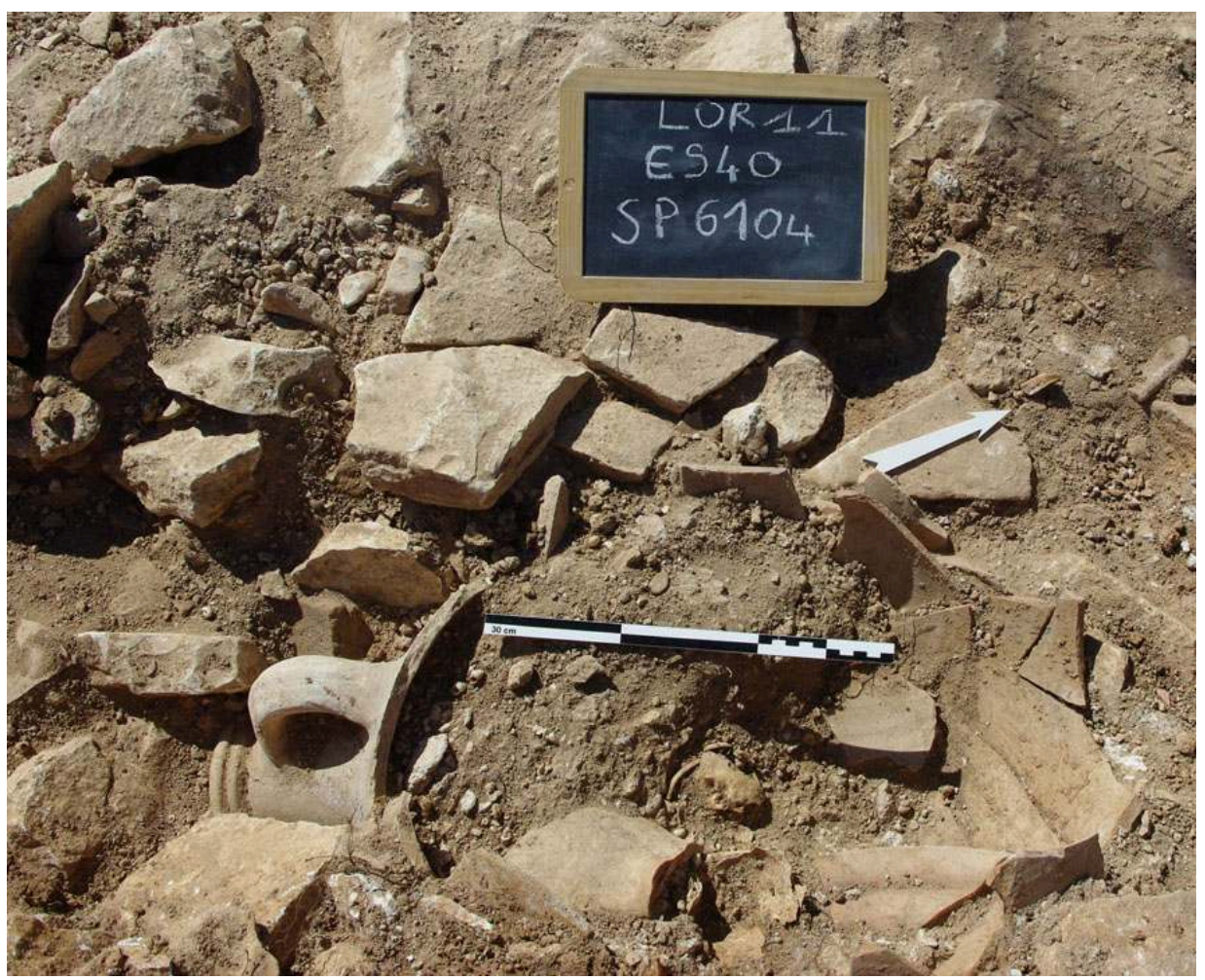

Cliché C. Rousse.

18 Les sépultures d'enfants en habitat ou dans des zones d'atelier céramique en activité sont bien connues en Gaule et plus largement dans le monde romain. Un des exemples les mieux documentés est l'ensemble funéraire découvert à Sallèles d'Aude, dans le bâtiment III de l'atelier ${ }^{4}$. À Loron, trois sépultures, dont une en amphore destinée à trois très jeunes enfants (IV $\mathrm{e}$. ap. J.-C.), avaient été déjà mises à jour par nos collègues italiens dans l'espace 36, en association avec de l'habitat.

Dans l'aile ouest, les sépultures de l'espace 40, plus tardives, montrent la continuité d'une occupation du site jusque dans la deuxième moitié du $\mathrm{V}^{\mathrm{e}} \mathrm{s}$. ap. J.-C., alors que la fonction artisanale du complexe était abandonnée (fin IV s. ap. J.-C.). On souhaiterait avoir plus d'éléments sur ces occupants tardifs du site, qui semblent avoir exploité comme carrière les structures architecturales de l'ancien atelier.

\section{Le préau de séchage (espace 48)}

$20 \mathrm{Au}$ nord de l'espace 40 se déploie une vaste salle en forme de $\mathrm{L}$, couvrant les $2 / 3 \mathrm{du}$ bâtiment occidental et plus d'1/3 de l'édifice nord (fig. 3). Délimité au nord et à l'ouest par les murs de façade de l'atelier, cet ensemble (espace 48) identifié comme un préau de séchage, mesure $38 \mathrm{~m}$ du nord au sud sur $30 \mathrm{~m}$ d'ouest en est, soit une superficie totale d'environ $920 \mathrm{~m}^{2}$. C'est, avec l'espace symétrique unissant l'aile nord et l'aile ouest (espace 49), la plus grande pièce de l'atelier (fig. 1).

21 En 2011, l'ouverture de deux sondages le long du mur de division interne MR 3230 a permis de retrouver deux imposantes bases de piliers rectangulaires $(1,40 \times 0,90 \mathrm{~m})$, orientée nord sud. Ces deux bases constituent le point de départ de deux travées de 
piliers $(1,10 \times 0,90 \mathrm{~m})$ qui divisent dans le sens nord-sud l'aile ouest de l'espace 48 (fig. 11). En 2007, deux travées de piliers orientées est-ouest avaient déjà été mises à jour à l'extrémité orientale de la pièce. La largeur des nefs est exactement identique : 5,2 $\mathrm{m}$ pour les nefs latérales; 4,8 $\mathrm{m}$ pour l'allée centrale. Le dégagement de l'ensemble des piliers a permis de vérifier l'adéquation parfaite des nefs nord-sud et est-ouest. Cette architecture interne répond à un plan précis, calculé et mis en œuvre avec soin pour faire coïncider les alignements sur des distances non négligeables $(38 \times 30 \mathrm{~m})$. Les constructeurs devaient également tenir compte de la déclivité du terrain et trouver des solutions architecturales pour contrecarrer la pression des murs et de la toiture. La présence d'imposants contreforts à l'extrémité sud des travées (en contrebas de la pente) et d'appui plus légers (lésènes) à l'extrémité ouest relève de ces contraintes. Elle suggère également que les piliers supportent un système d'arcades internes, sur lequel est posée la couverture, probablement un toit à double pente (proposition d'H. Broise CNRS / IRAA, que nous retenons ici). Enfin, les données stratigraphiques cohérentes des sondages réalisés à l'extrémité ouest (2007) et sud (2011) montrent que les bases de piliers et les murs sont, en l'état actuel, arasés au niveau de la fondation ou du sommet de celle-ci. Dans l'espace 49 , symétrique de l'espace 48 , nos collègues italiens ont mis à jour sur l'une des bases de piliers un départ d'élévation, dont les mesures $(90 \times 60 \mathrm{~cm})$ peuvent servir de référence pour restituer les piliers de l'espace 48 .

Fig. 11 - Loron. Architecture interne du préau de séchage, espace 48.

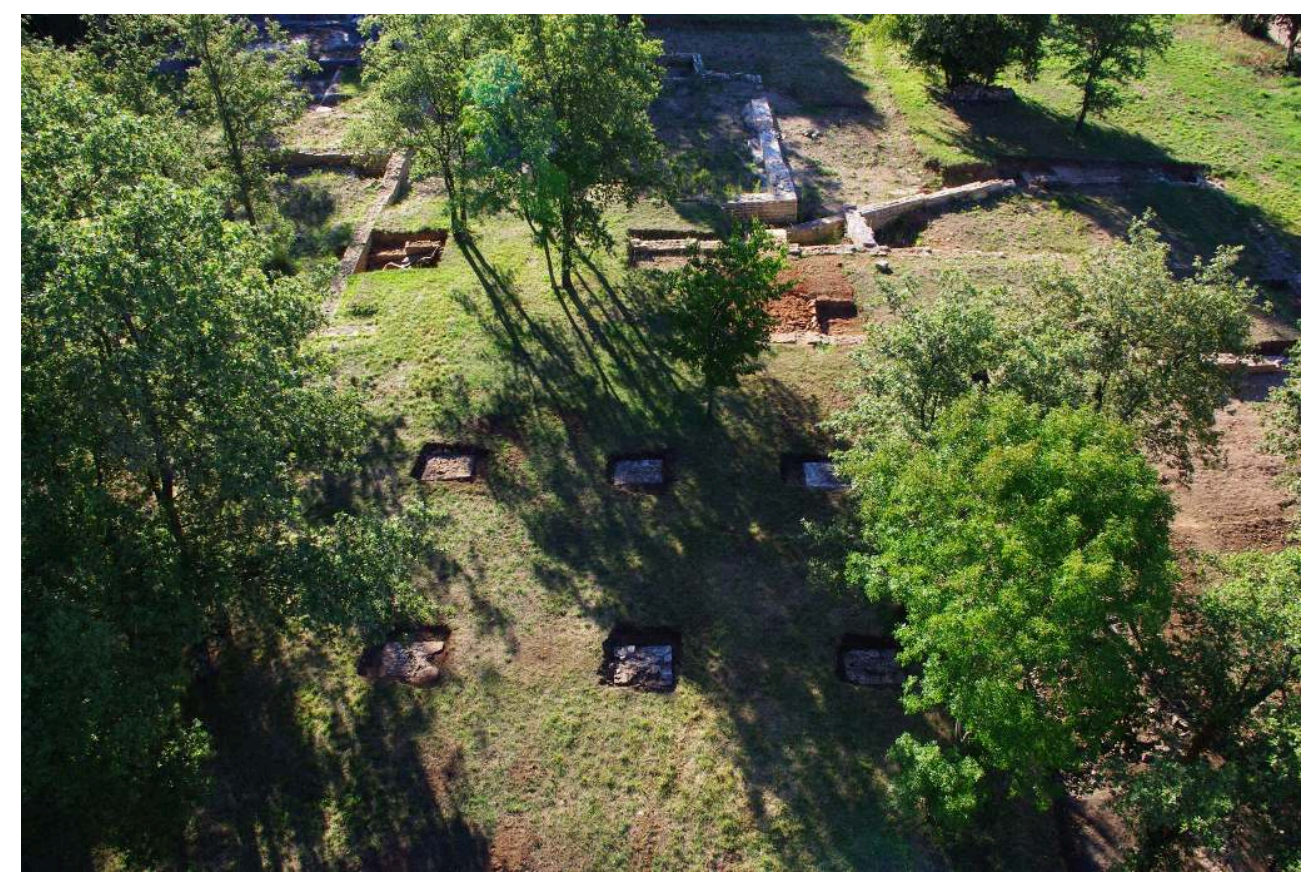

Cliché C. Taffetani, EFR.

Avec l'espace 48, les potiers de Loron disposaient d'une surface utile de $860 \mathrm{~m}^{2}$, une fois enlevé l'encombrement des murs et des piliers, soit $1600 \mathrm{~m}^{2}$, pour entreposer leurs productions, avec les deux préaux de séchage qui encadrent les fours à amphores (espaces 48 et 49). Cet ordre de grandeur donne la mesure de l'organisation fonctionnelle de l'atelier et reflète l'impératif de rendement qui préside à la construction ex nihilo du complexe. Le plan basilical adopté pour ces préaux de séchage est une solution classique à la couverture de larges espaces, que l'on retrouve dans la 
pars rustica des villas (celliers, chais) ou dans les espaces de séchage des ateliers de production céramique. Mais les dimensions des exemples connus (atelier d'Albinia en Toscane par exemple) sont bien inférieures à celles observées à Loron, soulignant le caractère hors norme de ce complexe de production istrien.

\section{Un nouveau relief ithyphallique dans l'espace 48}

Une autre surprise de la campagne 2011 a été de rencontrer un nouveau relief ithyphallique sculpté sur la paroi est du mur MR 3125. Le bas relief, représentant un phallus tourné vers le nord, occupe toute la face du bloc (fig. 12). Il s'agit probablement d'un jeu de maçon, puisque cette paroi du mur était recouverte par les niveaux de fondation supportant le plan de circulation dans l'espace 48. Ce phallus sculpté vient enrichir le corpus déjà connu à Loron, avec le bas relief au triple phallus et à l'étalon mis à jour en 2006 ; ce dernier était, au contraire, destiné à être vu dès l'entrée dans l'atelier. À la fin de la campagne 2011, le nouveau relief au phallus a été prélevé et déposé dans les réserves du musée de Poreč. Une copie a été commandée à un sculpteur local par V. Kovačić. Elle sera replacée in situ dans le courant de l'année 2011.

Fig. 12 - Loron. Espace 48, relief au phallus.

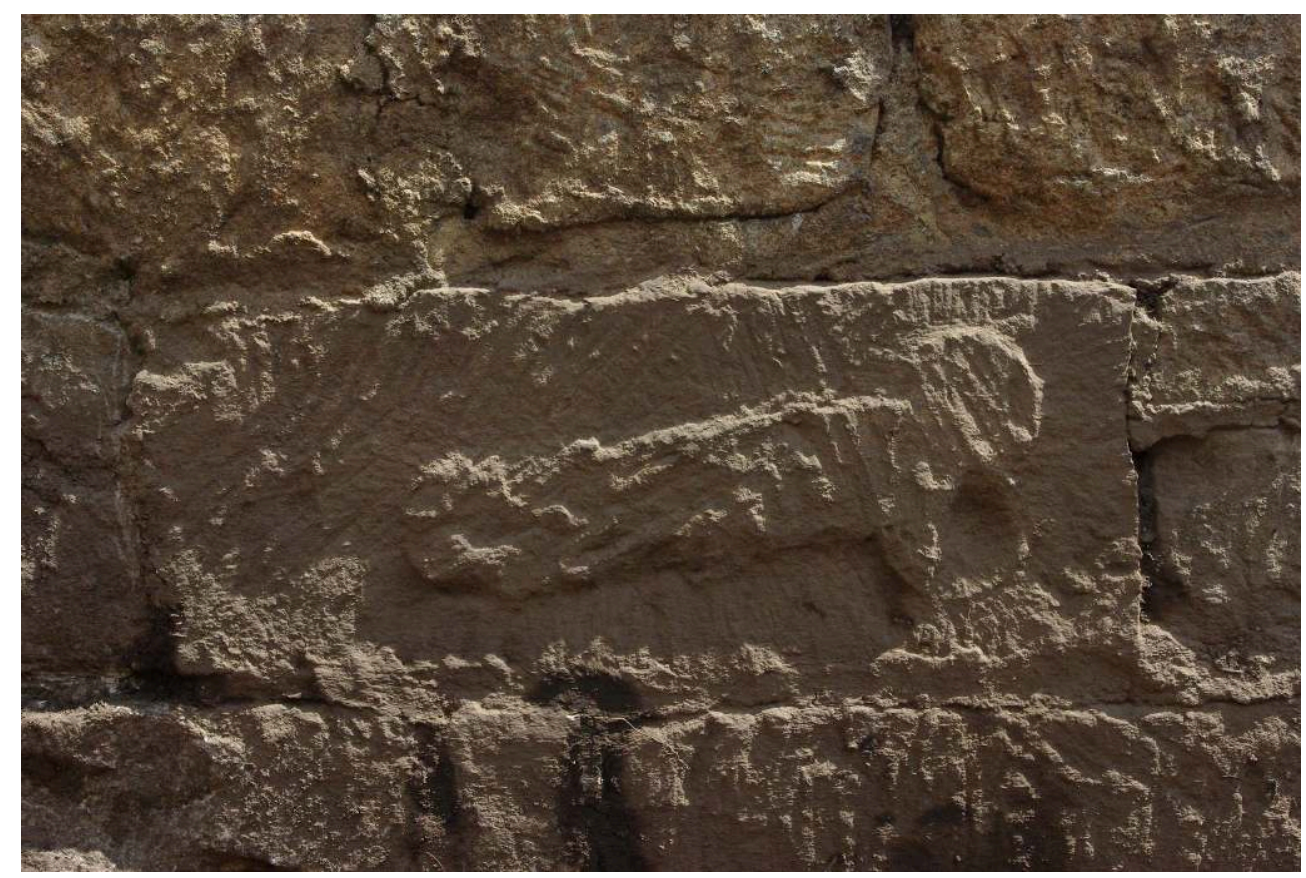

Cliché C. Rousse. 


\section{Prospections géophysiques et terrestres : de nouvelles perspectives de recherches sur le domaine maritime de Loron et son secteur résidentiel (villa maritime)}

Les prospections géophysiques et terrestres réalisées le long de la rive sud de la baie de Santa Marina mettent en évidence une occupation de type résidentiel, contemporaine de l'atelier de Loron (fig. 13). Vers le fond de la baie, des murs encore visibles perpendiculaires à la côte et se prolongeant dans la mer suggèrent la présence d'une plateforme artificielle, qu'il faut associer à la présence en retrait de la côte d'une citerne romaine encore bien conservée. À l'entrée de la baie, un sondage réalisé par A. Hanry (INRAP) témoigne également de cette occupation de type résidentiel. Il pourrait s'agir de la villa maritime des riches propriétaires de Loron, se développant le long de la côte nord du promontoire, dans un contexte maritime exceptionnel. Le potentiel archéologique de cette zone contraste en tout cas avec les résultats des prospections menées à l'intérieur du promontoire, qui se révèlent à l'inverse peu signifiants. La poursuite des prospections géophysiques et archéologiques devrait permettre de préciser le caractère résidentiel et aristocratique du secteur de Santa Marina et ses rapports topographiques et architecturaux avec l'atelier de Loron.

Fig. 13 - Loron. Prospections terrestres réalisées en 2011.

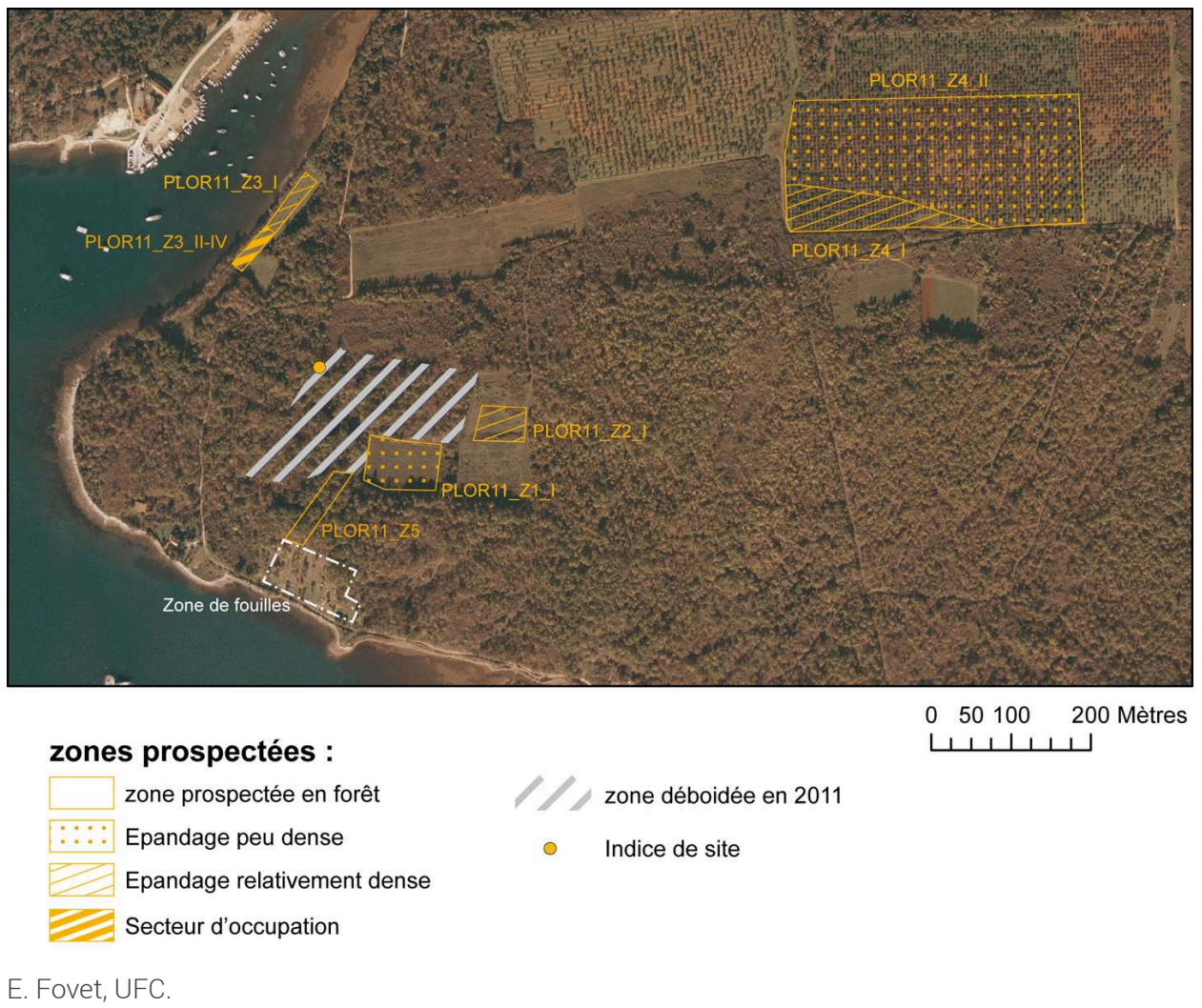




\section{BIBLIOGRAPHIE}

Bonifay et alii $2011=$ M. Bonifay, C. Capelli, M. Moliner, Amphores africaines de la basilique de la rue Malaval à Marseille ( $V^{e}$ siècle), dans Société française d'étude de la céramique antique en Gaule (SFECAG). Actes du Congrès d'Arles, Marseille, 2011, p. 235-254.

Duday et alii $1995=$ H. Duday, F. Laubenheimer, A.-M. Tillier, Sallèles d'Aude. Nouveaux nés et nourrissons gallo-romain, Besançon, 1995.

Žerjal $2010=\mathrm{T}$. Žerjal, Školarice near Koper. Some late roman contexts in the Northern Adriatic, dans S. Santoro, M. Pasquinucci, G. Guiducci (dir.), LRCW 3. Late Roman Coarse Wares. Comparison between western and eastern Mediterranean, Oxford, 2010, p. 703-710.

\section{NOTES}

1. MEFRA, 123-1, 2011.

2. Bonifay et alii 2011, fig. 11-12, n. 28.

3. Žerjal 2010, p. 703 et fig. 2.

4. Duday et alii 1995.

\section{INDEX}

Mots-clés : villa, atelier, amphore, stockage, sépulture, littoral, époque impériale institutions Centre Camille Jullian (Université Aix-Marseille / CNRS), École française de Rome Index géographique : Croatie, Loron

\section{AUTEURS}

\section{CORINNE ROUSSE}

Université Aix-Marseille - Centre Camille Jullian (UMR 7299) - corinne.rousse[at]gmail.com FRANCIS TASSAUX

Université Bordeaux 3 - Ausonius (UMR 5607) - francis.tassaux[at]u-bordeaux3.fr 